\title{
METODOLOGÍA PARA EVALUACIÓN DE RIESGO EN CAUCES PRÓXIMOS A LA SIERRA DE VALLEJO EN LA RIVIERA NAYARIT, MÉXICO: hidráulica, hidrología y territorio geográfico
}

\author{
RISK ASSESSMENT METHODOLOGY FOR NEXT CHANNELS IN THE \\ SIERRA DE VALLEJO IN RIVIERA NAYARIT, MEXICO: hydraulics, \\ hydrology and geographic territory
}

\section{METODOLOGIA PARA AVALIAÇÃO DE RISCOS EM CANAIS PRÓXIMOS À SERRA DE VALLEJO EM RIVIERA NAYARIT, MÉXICO: hidráulica, hidrologia e território geográfico}

\author{
J. Jesús Vázquez Magaña \\ Maestro en Ingeniería Hidráulica por la Universidad Autónoma de México \\ Estudiante del Doctorado en Ciencias Naturales y Biopsicosociales en el Instituto \\ de Estudios Superiores y Tecnológicos Matatipac \\ Profesor de Carrera en el Instituto Tecnológico de Tepic \\ Calzada del Ejército 98 W Tepic, Nayarit, México C.P. 63169, \\ E-mail: jesus_cnbps3g@institutomatatipac.edu.mx
}

María de Lourdes Vázquez Magaña Maestra en Restauración de Sitios y Monumentos por la Universidad de Guanajuato Estudiante del Doctorado en Ciencias Naturales y Biopsicosociales en el Instituto de Estudios Superiores y Tecnológicos Matatipac E-mail: arqrestauro@hotmail.com

Marcela Magallón Andalón

Maestra en Ciencias del Agua por la Universidad Autónoma del Estado de México- Facultad de Ingeniería

Estudiante del Doctorado en Ciencias Naturales y Biopsicosociales en el Instituto de Estudios Superiores y Tecnológicos Matatipac

E-mail: olmarmagand@yahoo.com.mx

\section{Eduardo Guerrero Flores}

Maestro en Administración Empresarial y Estudiante del Doctorado

en Ciencias Naturales y Biopsicosociales por el Instituto de Estudios Superiores y Tecnológicos Matatipac

E-mail: olmarmagand@yahoo.com.mx 


\title{
Resumen
}

En las diferentes etapas de desarrollo urbano de alguna región se debe priorizar la seguridad de las personas que la habitarán y de la infraestructura. En la formación académica del Ingeniero Civil, es importante conocer el comportamiento hidráulico e hidrológico de las zonas de edificación, con objeto de tener parámetros de diseño tanto de infraestructura pluvial, como de zonas de posible peligro por inundación, en el presente trabajo, se muestra un caso de estudio, mismo que pertenece al proyecto de investigación TEP-IC-2010-105, mismo que ha sido culminado el 28 de febrero de 2011, en los poblados ubicados en la Sierra de Vallejo, Nayarit, Hernández Sampieri, 1991, capitulo I.

Palabras Clave: Hidrología, hidráulica, urbanismo

\begin{abstract}
At different stages of urban development in any region should prioritize the safety of the people who inhabit and infrastructure. In the academic training of Civil Engineer, it is important to know the behavior of hydraulic and hydrological areas of building, in order to take both parameters infrastructure design storm, as areas of potential flood hazard, in this paper, is shown a case study, which belongs to the same research project TEP-IC-2010-105, it has been completed on 28 February 2011, in the villages located in the Sierra de Vallejo, Nayarit, Hernandez Sampieri, 1991, Chapter I.

Keywords: hydrology, hydraulics, urbanism
\end{abstract}

\section{Resumo}

Em diferentes estágios de desenvolvimento urbano em qualquer região deve priorizar a segurança das pessoas que habitam e infra-estrutura. $\mathrm{Na}$ formação acadêmica de Engenharia Civil, é importante conhecer o comportamento das áreas de hidráulica e hidrologia de construção, a fim de levar os dois parâmetros tempestade projeto de infraestrutura, como áreas de risco de inundação potencial, neste trabalho, é mostrado um estudo de caso, que pertence ao mesmo projeto de pesquisa TEP-IC-2010-105, foi concluído em 28 de Fevereiro de 2011, nas aldeias localizadas na Serra de Vallejo, Nayarit, Hernandez Sampieri, 1991, Capítulo I.

Palavras-chave: hidrologia, hidráulica, urbanismo

\section{Introducción}

La Sierra de Vallejo, ofrece una diversidad de combinaciones de tipo hidrológico e hidráulico, los cuales actúan con factores de seguridad y desarrollo urbano, dado que es en esta fracción del territorio nacional donde se ubica uno de los el polos de desarrollo turístico de México; "La Riviera Nayarit", con un crecimiento

Ateliê Geográfico Goiânia-GO v. 6, n. $4 \quad$ Dez/2012 $\quad$ p.5-20 $\quad$ Página 6


económico pujante, lo que induce la probabilidad de generar zonas de riesgo, dado el ritmo tan acelerado de crecimiento el cual en un momento dado puede dejar a un lado el análisis de estos aspectos; de igual forma, se hace imperiosa la necesidad de realizar propuestas que no antepongan el desarrollo urbano con respecto a la seguridad, por lo que se deberá efectuar una revisión de las propuestas en este rubro, que permitan vislumbrar un desarrollo sustentable en la zona poblada y cercana al Delta del Río Ameca Figuras 1 y 2.

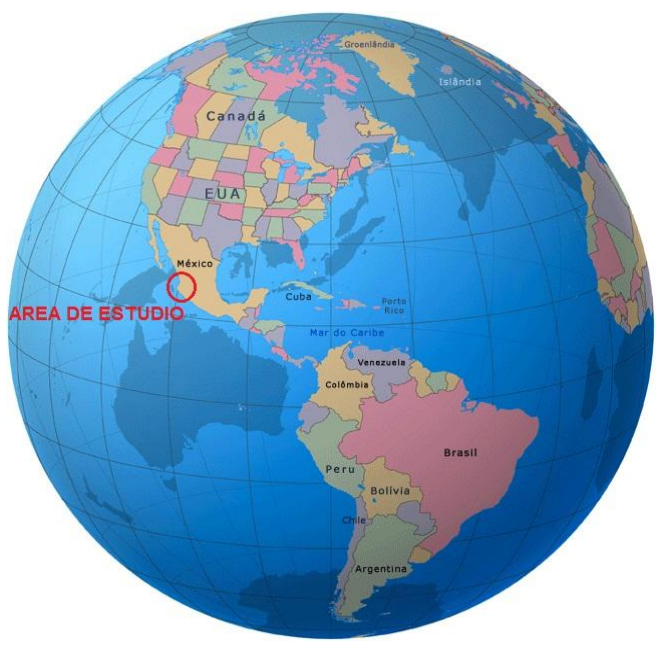

Figura 1: Ubicación área de estudio

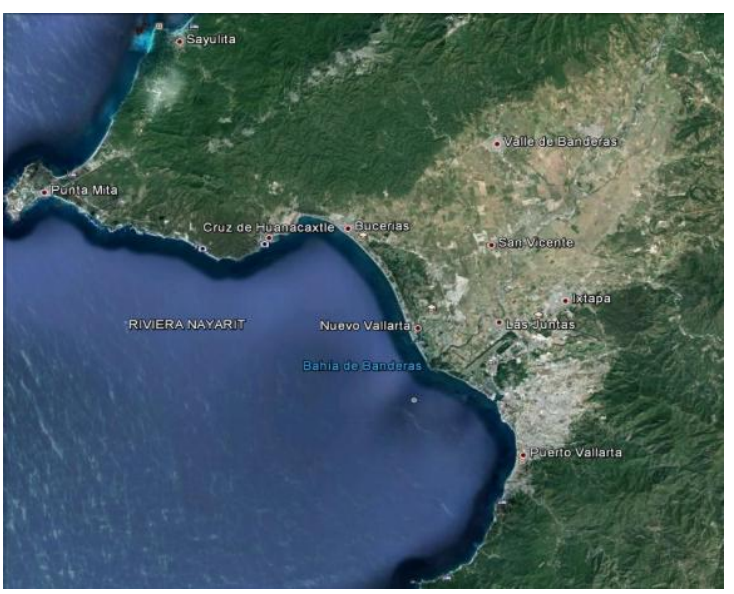

Figura 2: Delta Río Ameca Riviera Nayarit.

Lo anteriormente expuesto demanda aplicar modelos hidrológicos e hidráulicos tanto en la cuenca del Río Ameca como en la Sierra de Vallejo, territorio que forma parte de la Riviera Nayarit, mismos que sean sustentados con base en el análisis estadístico de toda la información hidrométrica y climática existente, tomando en cuenta los desarrollos de métodos directos e indirectos (APARICIO, 2006, Pa.213 y 239), la Sierra en cuestión, tiene un desarrollo de cuencas que aportan escurrimientos al delta del Río Ameca en un área de 2,000 $\mathrm{Km}^{2}$, la distancia del recorrido del Río Ameca desde su nacimiento hasta su desembocadura es de aproximadamente $240 \mathrm{~km}$, y dicha Sierra aporta agua en una longitud de $40 \mathrm{Km}$, comprendiendo la región hidrológica Ameca, misma que aproximadamente conforma el $25 \%$ de los escurrimientos de la frontera Sur del Estado de Nayarit $\left(3,000 \mathrm{~km}^{2}\right)$, el río Ameca tiene su origen a $25 \mathrm{~km}$. al Oeste de la Ciudad de Guadalajara, en la parte más elevada de la Cuenca, a 2,000 m.s.n.m. Desde un punto situado a $43 \mathrm{~km}$. aguas abajo del Puente Ameca y hasta su desembocadura, el Río Ameca es límite de los estados de Nayarit por su margen derecha y Jalisco por su 
margen izquierda. La Sierra de Vallejo por su parte, cuenta en sus cuencas con una precipitación media anual de 1,200 mm. Al carecer de estaciones hidrométricas su análisis se deben fundamentar en datos de precipitación, misma que tiene un valor medio de 1,600 $\mathrm{mm}$. La hidráulica de todas las subcuencas que pertenecen a dicha región es la propia de una cuenca con baja pendiente con tres grados de libertad, con coeficientes de rugosidad que oscilan entre 0.035 y 0.045 (SOTELO, 1974 p. 111), predominando gravas y arenas, así como rocas de tamaño superior a los $50 \mathrm{~cm}$, por lo que es factible la construcción de modelos hidráulicos a partir de información topo batimétrica y con referencia Proyecto de Investigación PITT-IT-02/2009-006.

Para el Instituto Nacional de Geografía Estadística e Informática INEGI (2011) esta zona comprende en su totalidad, el municipio No. 20 del estado de Nayarit Bahía de Banderas, los ejidos; San Juan de Abajo, Colomos, El Porvenir, San Vicente, Mezcales y Jarretaderas, tienen colindancia con el Río Ameca, formando parte de la Riviera Nayarit, existen planes de ordenamiento urbano, los cuales deben contemplar el aspecto de seguridad ante los cambios en el régimen hidráulico de las corrientes, que inciden en dichas poblaciones, el presente trabajo pretende mostrar una metodología que caracterice a la región y que a partir de modelos hidrológicos empíricos permita establecer parámetros de seguridad que a su vez sirvan para mejorar la imagen urbana de los mismos.

\section{Metodología}

El empleo de la Probabilidad y Estadística aplicadas directamente a la hidrología, hacen es posible que se efectúe el análisis de datos de alguna cuenca con seguridad y una buena aproximación a la tendencia real, de los fenómenos climáticos, es por ello que en este apartado se describen los métodos usados, para determinar las avenidas y tormentas de diseño. Los métodos usados para dichos análisis deben contemplar datos de precipitación de estaciones climatológicas aledañas a las subcuencas estudiadas (método indirecto). Con ello se obtiene una asociación de Gastos a Periodos de Retorno a partir de datos de lluvia (APARICIO, 2006, p. 239), dichos trabajos empleados como parámetros de simulación hidráulica (SOTELO, 2006, p. 25 y LEVI, 1993), permiten conocer los niveles que alcanzan avenidas en sitios próximos a 
las poblaciones de la región, con el fin de presentar propuestas geométricas fundadas en el análisis de ingeniería, los métodos de hidrología citados se describirán a continuación:

\section{Hidrología, Tormentas Y Avenidas De Diseño}

Función de densidad de probabilidad $f(x)$

Se define como función de densidad de probabilidades, al modelo matemático de la variable aleatoria continua (precipitación o gastos), es decir, la curva limite del histograma cuando tiene intervalos infinitos de clase que posee las siguientes propiedades (APARICIO, 2006, p. 239):

ـ $\quad f(x) \geq 0$; la curva es positiva

․ $\int_{-\alpha}^{+\alpha} f(x) d x=1$; el área bajo la curva es igual a uno

a $\int_{a}^{b} f(x) d x=P(a \leq x \leq b)$; a y b son valores cualesquiera de $\mathrm{x}$, con la condición $\mathrm{a}<\mathrm{b}$

Función de distribución de probabilidad F(x)

Esta es asociada a una variable aleatoria $X$, que puede tomar valores reales, como la probabilidad de que dicha variable tome valores menores o iguales a un valor dado $\mathrm{x}$, para toda $\mathrm{X}$ real, esto se expresa:

$$
F(x)=P(X \leq x)
$$

Donde X y $\mathrm{x} \in \mathrm{R}$

Sus principales características son: 


$$
\begin{array}{ll}
\text { ป } & F(\infty)=1 \\
\text { ป } & F(-\infty)=0 \\
\text { ป } & F(x+\Delta x) \geq F(x) \text { si } \Delta x \geq 0 \\
\text { ป } & F(a \leq x \leq b)=f(b)-f(a)
\end{array}
$$

La demostración matemática se centra en que $\mathrm{f}(\mathrm{x})$ es la derivada de $\mathrm{F}(\mathrm{x})$ :

$$
f(x)=\frac{d F(x)}{d x}
$$

En problemas hidrológicos se deberán definir valores extrapolados que excedan los límites del conjunto de datos $\mathrm{X}$ analizados por lo que se define y adopta el concepto de periodo de retorno $\mathrm{Tr}$, el cual es el tiempo promedio que se requiere para que la variable en cuestión exceda un determinado valor x.

Para una muestra analizada y ordenada por datos en forma anual, es posible plantear la siguiente ecuación basada en el concepto de probabilidad definido por:

$$
P(X \geq x)=\frac{m}{n}
$$

Donde $\mathrm{m}$ es el orden o frecuencia del evento $\mathrm{x}, \mathrm{y}$ n es el número de eventos de $\mathrm{x}$. Por lo tanto se tiene:

$$
P(X \geq x)=\frac{1}{T r}
$$

La ecuación anterior indica que si el evento hidrológico $\mathrm{X}$ igual o mayor que $\mathrm{x}$, ocurre una vez en $\operatorname{Tr}$ años, entonces las probabilidades de excedencia $X>=x$ y la no excedencia $\mathrm{X}<=\mathrm{X}$, se da por la igualdad manifestada a continuación:

$$
\operatorname{Tr}=\frac{1}{P(X \geq x)}=\frac{1}{1-P(X \leq x)}
$$

Por lo tanto se tiene que:

$$
\operatorname{Tr}=\frac{n+1}{m}
$$

Métodos estadísticos y/o probabilísticos.- Los métodos Estadísticos permiten estimar la avenida máxima de un río, a partir de la información hidrométrica, es decir de 
la serie de avenidas máximas anules registrada. Lo anterior, en base de la exploración de la Función de Distribución de Probabilidades que tiene la variable , en este caso, Gasto Máximo Anual y precipitaciones máximas anuales para el caso del afluente.

En la actualidad se disponen de muchas Funciones de Distribución de Probabilidades y las que se aplican generalmente en fenómenos hidrológicos, las cuales se pueden dividir en tres grandes grupos (APARICIO, 2006, p. 239):

1. Distribuciones de valores medios y muestras grandes (número de datos mayor a 30 años), con tendencias aproximadas a una distribución Normal o tipo Gauss. Aplicadas principalmente a variables como, temperatura, presión, etc.

2. Distribuciones para muestras pequeñas (número de datos menor a 30 años), como son la Ji cuadrada de Pearson, t de student, F de Fisher.

3. Distribución para valores extremos, por ejemplo, los gastos máximos anuales de cierta corriente, las precipitaciones máximas anuales registradas en 24 horas, etc.

Las distribuciones del tercer grupo son las más usuales dados los registros con los que contamos en nuestro país, y son los denominados métodos estadísticos para determinar avenidas de diseño para diferentes períodos de retorno. Las funciones más usadas para el fin antes descrito se enumeran a continuación:
a) Gumbel de valores Extremos
b) Doble Gumbel
c) Log Normal
d) Log Pearson III
e) Gamma

La función Gumbel se desarrolló para el análisis de los valores extremos de muestras o series hidrológicas, y se expresa de la siguiente manera (STREETER WYLIE, 1998, p. 62):

$$
F(x)=e^{-e^{-\alpha(x-\beta)}}
$$


Teniendo en cuenta la función de densidad de probabilidad es entonces:

$$
f(x)=\alpha e^{\left[-\alpha(x-\beta)-e^{-\alpha(x-\beta)}\right]}
$$

Donde $\alpha$ y $\beta$ son los parámetros de la función que pueden ser obtenidos a través de momentos, mínimos cuadrados y máxima verosimilitud (APARICIO, 2006, p. 239).

La función Doble Gumbel, es empleada en muchos lugares, especialmente en zonas costeras, los eventos máximos anuales pertenecen a 2 poblaciones diferentes. La primera es la de los eventos producidos por precipitaciones relacionadas con los fenómenos metereológicos dominantes en la región es estudio y la segunda es la de los eventos producidos por precipitaciones ciclónicas normalmente mayores que las primeras.

Se ha demostrado que en estos casos la función de distribución de probabilidad se puede expresar de la siguiente manera, (APARICIO, 2006, p. 250):

$$
F(x)=F_{1}(x)\left[p+(1-p) F_{2}(x)\right]
$$

donde:

p, probabilidad de lluvia de temporada.

F1(x), precipitaciones ciclónicas

$\mathrm{F} 2(\mathrm{x})$, lluvias de temporal

El número de parámetros de la función son :

n1, número de parámetros de F1(x)

n2, número de parámetros de F2(x)

El parámetro restante es $\mathrm{p}$. 
La estimación de $\mathrm{p}$, define la probabilidad de que un año cualquiera sea no ciclónico, la propuesta es (APARICIO, 2006, p. 239):

$$
p=\frac{N n}{N_{T}}
$$

Donde:

Nn : es el número de años de registro en que el gasto máximo no se produjo por una tormenta ciclónica.

NT : es el número total de años de registro.

Es común aceptar que $\mathrm{F} 1(\mathrm{x})$ y $\mathrm{F} 2(\mathrm{x})$ son del tipo Gumbel por lo que de ser así, entonces:

$$
F(x)=e^{-e^{-\alpha_{1}\left(x-\beta_{1}\right)}}\left[p+(1-p) e^{-e^{-\alpha_{2}\left(x-\beta_{2}\right)}}\right]
$$

Donde, $\alpha_{1}$ y $\beta_{1}$ son los parámetros correspondientes a la primera población y $\alpha_{2} \quad y \quad \beta_{2}$ corresponden a la segunda población.

El uso de cada función de distribución de probabilidades, quedará sujeta al criterio del hidrólogo y/o aquella distribución que muestre el mejor ajuste de acuerdo al análisis del mínimo error cuadrático, haciendo notar que es muy importante precisar la asociación de gastos a periodos de retorno empleando la metodología descrita con antelación.

Para este caso se emplea el análisis de estación hidrométrica de Gaviotas y La Desembocada, destacando que el empleo de datos hidrométricos nos ofrece una mayor aproximación a eventos naturales, el análisis de lluvia escurrimiento (FUENTES, 2008, p.60) se deberá precisar en trabajos posteriores para las cuencas que provienen de la Sierra de Vallejo y que inciden en las poblaciones estudiadas. 


\section{Hidráulica}

La hidráulica de las corrientes alimentada con el empleo de topografía, batimetría, aerofotogrametría y los resultados hidrológicos previos, permiten definir la simulación hidráulica con el objeto de conocer el comportamiento del Río Ameca y su influencia con los centros de población.

Como primer término se tiene que para cualquier análisis hidráulico es necesario definir las ecuaciones a usar que par el caso son las siguientes (SOTELO, 2006, p.55) (CHOW, 1994, p.75).

Ecuación de continuidad

$$
\frac{\partial A}{\partial t}+\frac{\partial Q}{\partial x}=0
$$

Ecuación de cantidad de movimiento

$$
\frac{\partial Q}{\partial t}+\frac{\partial}{\partial x}\left(\frac{Q^{2}}{A}+g I_{1}\right)=g A\left(S_{o}-S_{f}\right)+g I_{2}
$$

Las ecuaciones descritas anteriormente son las llamadas ecuaciones de SaintVenant, de las que su desarrollo tiene su fundamento en las siguientes hipótesis:

Flujo unidimensional: la velocidad es uniforme en la sección transversal, además el nivel del agua en dirección normal al flujo es horizontal.

La curvatura de las líneas de corriente y las aceleraciones verticales son pequeñas, la distribución de presiones, por tanto, es hidrostática.

Pendiente pequeña: la pendiente del fondo del cauce es pequeña.

Pérdidas de energía: semejantes a las del flujo uniforme, esto implica que es válido utilizar cualquiera de las fórmulas para flujo uniforme.

La densidad del agua es constante.

Dichas ecuaciones tienen solución analítica en algunos casos, por tratarse de ecuaciones diferenciales parciales en general deben resolverse con métodos numéricos. Para las ecuaciones del escurrimiento en cauces, el método que más ventajas tiene es el 
de diferencias finitas, pues además de su relativa sencillez, da resultados suficientemente precisos (MARTIN, 2009, p.45) (MAZA, 1987, p. 3.1).

Existe actualmente para la obtención de perfiles el programa de computo denominado Hec-Ras con diferentes versiones, mismo que es desarrollado por el cuerpo de Ingenieros de la Armada de los Estados Unidos.

El modelo usado en la definición de los perfiles sigue principalmente la solución de la ecuación de la energía por tramos en los que se ha dividió el río, esta ecuación es la siguiente (I) (SOTELO, 1974, p.121 ):

$$
Z_{1}+Y_{1}+\alpha_{1} \frac{V_{1}^{2}}{2 g}=Z_{2}+Y_{2}+\alpha_{2} \frac{V_{2}^{2}}{2 g}+S F
$$

al tratar la ecuación (I) en función del gasto se tiene:

$$
Z_{1}+Y_{1}+\alpha_{1} \frac{Q_{1}^{2}}{2 A_{1}^{2} g}=Z_{2}+Y_{2}+\alpha_{2} \frac{Q_{2}^{2}}{2 A_{2}^{2} g}+S F
$$

la ecuación (II) se resuelve para cada uno de los tramos considerando las pérdidas de fricción estimadas por medio de la ecuación de Manning para este caso se emplean valores de 0.035 y 0.045, (CHOW, 1994, p. 75).

Es de hacer notar que en el presente trabajo se empleó información meteorológica de la Comisión Nacional del Agua, Dirección Local en Nayarit, así como topografía planteada en el vuelo Aerofotogramétrico de 2010 efectuado por Gobierno del estado de Nayarit, información que es del dominio público, distribuida por el Instituto Nacional de Geografía Estadística e Informática INEGI (2011), además de la realización de estudio de batimetría en puntos estratégicos de una cuenca de la Sierra de Vallejo, que descarga sus aguas en el Río Ameca, específicamente se contempla el arroyo La Quebrada, con la finalidad de complementar el modelo de altimetría y agrimensura necesario para la construcción de un modelo hidráulico, la modelación hidráulica tendrá como objetivo primordial, identificar los niveles del agua que se alcanzan en este arroyo, asociados a diversos periodos de retorno y con condiciones de frontera definidos por la avenida de diseño calculada para dicha corriente (APARICIO, 2006, p.209). 


\section{Desarrollo Urbano}

El análisis se centra en orientar un desarrollo urbano hacia zonas más seguras, de acuerdo con el Plan Municipal de Desarrollo Urbano de Bahía de Banderas, Estado de Nayarit, México (2008 - 2011, p. 89), al considerar el comportamiento hidráulico e hidrológico del arroyo La Quebrada, para realizar un estudio del entorno urbano afectado, que permita restringir el uso del suelo de acuerdo a necesidades y seguridad.

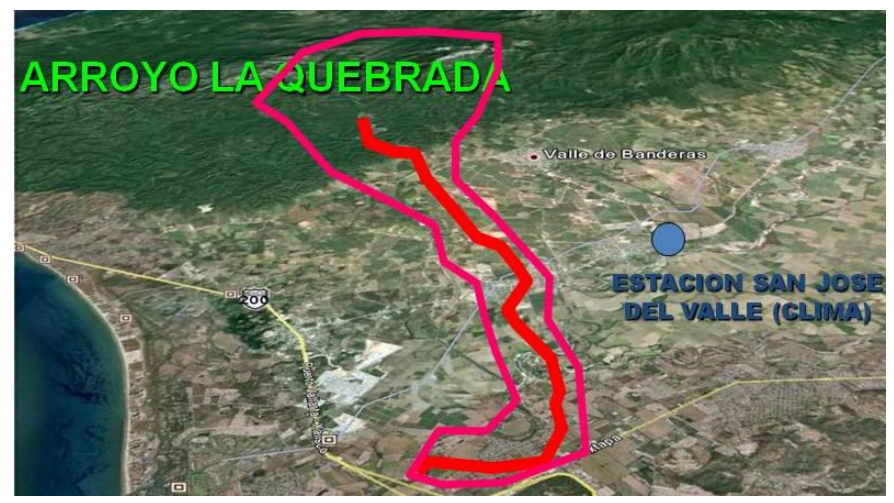

Figura 3: Cuenca analizada

\section{Resultados Y Discusión}

En primer término se presenta el análisis hidrológico planteado desde un inicio considerando datos climatológicos de la estación denominada San José del Valle, ubicada en la localidad del mismo nombre, tal y como se muestra en la figura número 3 superior. De esta parte de la investigación se desprenden resultados que representan gastos asociados a diversos periodos de retorno, dentro de los cuales el recomendado según normatividad de la Comisión Nacional del Agua (CONAGUA, 2007), Órgano desconcentrado de la Secretaría del Medio Ambiente y Recursos Naturales, del Gobierno de los Estados Unidos Mexicanos, recomienda que para diseño de obras de protección y encauzamiento es el de $\mathbf{1 0 0 0}$ años, el cual arroja un gasto coincidente de $257.81 \mathrm{~m}^{3} / \mathrm{s}$, aplicable al modelo hidráulico que se conforma con 117 secciones transversales, y que tiene una coincidencia con resultados obtenidos a partir de la determinación de gastos por el método de sección pendiente, obtenido durante la 
Tormenta Norman en el 2011, $259.00 \mathrm{~m}^{3} / \mathrm{s}$, como se aprecia en la Figura 4, con lo que se determina que el modelo hidrológico es adecuado, para los hidrográmas calculados Figura 5.

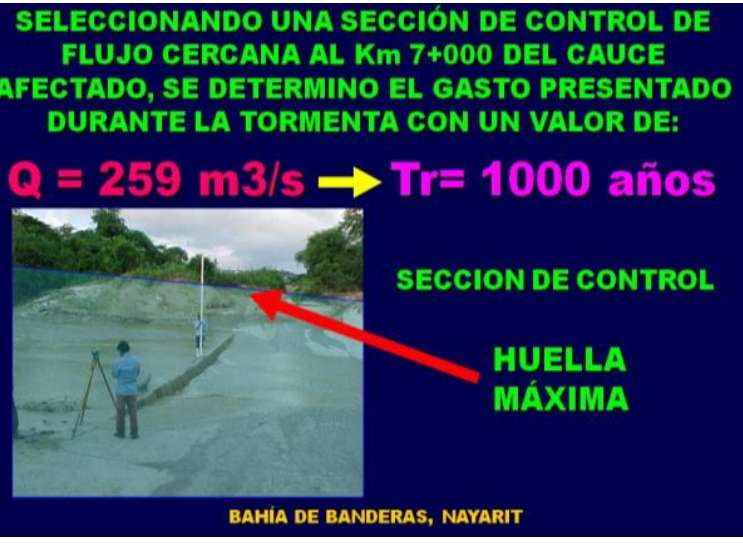

Figura 4: Sección de control analizada

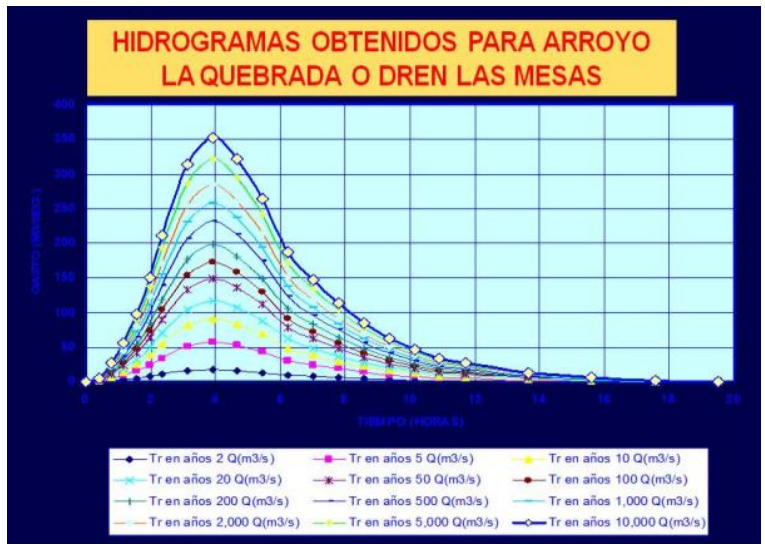

Figura 5: Hidrogramas obtenidos

En segundo término se construye modelo hidráulico con información topográfica con curvas de nivel a cada $0.50 \mathrm{~cm}$. Lo anterior con la finalidad de determinar los sitios de desbordamiento del rio y los niveles máximos que este alcanza a la altura de la localidad de San José del Valle, en Nayarit, todo ello a partir de datos de aerofotogrametría reconstituida y distribuida por el Instituto Nacional de Geografía Estadística e Informática INEGI (2011) y batimetría levantada en sitios estratégicos (2011) utilizando el gasto manifestado anteriormente, y un perfil asociado a periodo de retorno de 1000 años, del cual con el análisis del modelo, se desprende que el arroyo La Quebrada en las condiciones actuales, soporta el gasto de $259 \mathrm{~m} 3 / \mathrm{s}$ asociado a un $\mathrm{Tr}-$ 1000 con desbordamientos en tres sitios, sin embargo, se podrían considerar como aberturas de inundación que pueden ser cubiertas con la construcción de tramos de bordos con una longitud de $200.00 \mathrm{~m}$, es recomendable analizar la estabilidad estructural de los bordos naturales (MAZA, 1987, p. 3.1) que protegen a la comunidad de San José del Valle, municipio de Bahía de Banderas, Nayarit.

Con fundamento en el análisis hidrológico e hidráulico citado con antelación, se analiza la influencia respecto a centros de población colindantes con corrientes en la Sierra de Vallejo y se hace posible que sean planteadas opciones de crecimiento y solución para riesgos de inundación, y se vislumbra que la metodología empleada es la adecuada. 
Estrategias Generales: Se pretende llevar a cabo esta estrategia a las localidades afectadas, cuyos aspectos específicos involucran a cada localidad de la Sierra de Vallejo, mediante el planteamiento siguiente:

1. Un crecimiento ordenado, con un aprovechamiento adecuado del suelo.

2. Definir claramente los límites a cada localidad afectada, incluyendo la prevención de las áreas de reserva urbana suficiente y adecuada para el crecimiento esperado, fuera de estos límites no deberá permitirse ninguna acción urbanística (SCHUSTER, 1943, p.45).

3. Impulsar el establecimiento de industrias agropecuarias y de los servicios correspondientes de acopio y comercialización, mediante la previsión de reservas urbanas adecuadas.

4. Definir la localización de corredores de usos mixtos de nivel barrial que permitan el establecimiento ordenado de comercios y servicios básicos y del equipamiento urbano requerido para la atención de las necesidades.

5. Conservar los elementos de valor patrimonial y de interés paisajísticos pudiendo ser utilizados como una atracción para el turismo.

\section{Estrategias Para Las Localidades De San José Del Valle, El Porvenir Y}

\section{San Vicente:}

Al igual que para las otras localidades, se propone un bordo ubicado al Suroeste de las localidades de San José del Valle; El Porvenir y San Vicente.

Se propone la reubicación de vivienda al Norte de la localidad de San José del Valle, con una superficie de 3.40 ha. La reubicación de viviendas de la localidad de El Porvenir se propone al Noroeste de la localidad con una superficie de 3.47 ha. 
Se propone la continuidad del bordo a lo largo de estas tres localidades, con un diseño arquitectónico paisajista.

Se propone la rehabilitación de la avenida principal (Constitución) ya que esta es la vialidad que comunica a todas las localidades afectadas ya mencionadas.

Se propone manejar el área de comercio en la vialidad que comunica El Porvenir y San José del Valle, la propuesta de proyecto; una ciclo pista integrando las tres localidades para un mejor desplazamiento de sus habitantes y mayor seguridad, así como andadores y jardines.

\section{Conclusiones}

Es importante definir que los resultados y anexos reportados en el presente proyecto de investigación TEP-IC-2010-105, se presentan en tres apartados, los cuales siguen el orden definido para cada uno de la siguiente forma: análisis hidrológico, hidráulico y desarrollo urbano, mismos que forman parte de los bienes documentales del Instituto Tecnológico de Tepic y del Instituto de Estudios Superiores y Tecnológicos Matatipac, es importante remarcar que con estos resultados se cierra una investigación que contempla tanto el funcionamiento del Río Ameca como en cada localidad ubicada en el Delta del Río Ameca, dado que el resultado final arroja que existen localidades que tienen situaciones de peligro de inundación dadas las divagaciones fluviales, tanto en Nayarit como en Jalisco, en la Riviera Nayarit, lo que compromete a los tres niveles de Gobierno, que existen en México, a poner más atención en la realización de obras que permitan tanto la disminución de riesgos de inundación y divagación en la trayectoria de la corriente, así como en el aspecto relevante de la imagen urbana.

Es de hacer notar que la metodología propuesta y los trabajos realizados de mediciones geométricas y estadísticas, a las corrientes torrenciales y continuas, son aplicables para la evaluación de riesgos esta Región, situación que el Desarrollo Urbano, deberá emplear para la evaluación de crecimiento futuro y actual.

\section{Referencias}

HERNÁNDEZ, Sampieri, Roberto. Metodología de la Investigación. México: McGraw-Hill, 1991. 15 páginas. 
APARICIO MIJARES, Francisco Javier. Fundamentos de Hidrología de Superficie. México: LIMUSA, 2006. 239 -291 páginas.

SOTELO ÁVILA, Gilberto. Hidráulica General. México, LIMUSA, 1974. 111 - 183 Páginas.

SOTELO ÁVILA, Gilberto. Hidráulica de canales II. México: Facultad de Ingeniería de la UNAM, 2006. 55 página.

LEVI, Enzo. Tratado Elemental de Hidráulica. México: Instituto Mexicano de Tecnología del Agua (IMTA), 1993. 25-50 páginas.

FUENTES MARILES, Oscar. Estudio Hidrológico para Obras de Protección. México: UNAM, 2008. 60 páginas.

MARTÍN VIDE, Juan P. Ingeniería Fluvial. Cataluña: Universidad Politécnica de Cataluña, España, 2009. 45 páginas.

STREETER, Victor L. Wylie E. Benjamin. Mecánica de Fluidos. EEUU: Mc Gray Hill, 1998, 62 páginas.

CHOW, Ven Te. Hidráulica de Canales. EEUU: Mc Gray Hill, 1994. 75 páginas.

MAZA ÁLVAREZ, José Antonio. Introduction to River Engineering. Perugia: Universitá Italiana, per Stranieri, Italia, 1987. 31 páginas.

SCHUSTER ISIDORO, Víctor. Urbanismo. España: El Ateneo, 1943. 45 páginas.

GOBIERNO MUNICIPAL, Bahía de Banderas Nayarit. Plan Municipal de Desarrollo Urbano de Bahía de Banderas, Municipio de Bahía de Banderas: Gobierno municipal de Bahía de Banderas, Nayarit, México, 2011. 89 páginas.

GOBIERNO FEDERAL, México. Bases Meteorológicas. México: Comisión Nacional del Agua Dirección Local en Nayarit, 2011. 1-120 páginas.

GOBIERNO FEDERAL, México. Información cartográfica del Estado de Nayarit. México: Instituto Nacional de Geografía Estadística e Informática INEGI, 2011. 1-200 páginas.

Recebido para publicação em setembro de 2012 Aprovado para publicação em outubro de 2012 